\title{
TEXTURE PARAMETRIZATION METHOD FOR IMAGE SEGMENTATION
}

\author{
A. Casals, J. Amat and A. Grau \\ Dept. Enginyeria de Sistemes, Automàtica i Informàtica Industrial \\ Universitat Politècnica de Catalunya \\ c/ Pau Gargallo no 5, 08028 Barcelona (Spain)
}

\begin{abstract}
In this paper we define six parameters addressed to parametrize the texture characteristics of an image towards its segmentation. With the aim to operate at high speed, these parameters have been defined looking for an acceptable compromise between discrimination capacity and easyness to implement a specific architecture for them.
\end{abstract}

\section{Basic Textures Considered}

Texture is a qualitative concept of images and so, its parametrization is a difficult task. The ways followed to obtain a quantification for image texture have been, on one hand, based on a structural analysis [Tsu],[Mat], which analyze the regularity and uniformity of the image intensity, while other methods are based on statistical analysis of the local properties that occur repeatedly throughout the image [Har], [Bou]. Zucker [Zuc] proposes a model based on these two approaches, while in [Shi] a study is done about the human texture visual field.

The work presented on texture parametrization has as a goal obtaining the most discriminating vector, in view to segmentation from the observable texture of the different parts or objects that appear on the scene, and looking for a compromise between the attainable efficiency and the easy of obtaining, by means of a high speed specific hardware, the quantifiable values that define texture.

In order to attain a certain discrimination capability of the texture functions $T$, complementary to other functions such as color, it is necessary to define a given number of parameters $\tau_{i}$ quantified with a sufficient resolution to reliable differentiate the textures of an image. In a previous work, only a subset of the proposed parameters has been used to segmentate images in some specific environments [Cas].

These parameters don't seek to be more discriminant than others described by numerous authors, that in most cases require a higher level of data processing [Man], [Buf], and which are used in applications that don't present computing time restrictions such as terrain classification, satellite imagery, etc. What we pretend is to obtain these description parameters at high speed for applications that need real time operation.

The vector of characteristics we use is based on the following parameters:

Blurriness. A region with this texture presents soft intensity changes in any direction. Granularity. This parameter quantifies the presence of a high density of non concatenated gradients in a given region.

Discontinuity. This parameter measures the number of lines discontinuities. Abruptness. Abruptness measures sudden changes of direction of the lines of a region. Straightness. This parameter indicates the density of straight lines in a region. Curviness. This parameter measures the density of non straight lines in a region.

The research described in this paper has been supported partially by SKIDS (ESPRIT - 1560) 
Fig. 1 shows the affinities between different textures clearly defined and the six texture parameters considered.

With these six parameters which are not mutually excluding, it is defined a texture vector $\mathrm{T}\left[\tau_{1}, \ldots \tau_{6}\right]$, which quantifies the degree of affinity of every region of an image to each one of these parameters.

These selected parameters are not essentially different from some of the classic ones utilized such as: Coarseness, directionality, regularity, .... On the other hand, the natural texture patters defined by Brodatz [Bro] are also defined from these parameters, some of them can be defined in terms of exclusively one parameter, while others need up to three different ones.

The use of these parameters, as they are defined, don't provide information such as: regularity/irregularity, or symmetry/asymmetry, which correspond to a more global scope that surpass the region evaluated and that has to be obtained in a higher processing level.

Once the six texture parameters have been obtained for every pixel of the image, tacking into account its $8 \times 8$ pixels environment, a new lower resolution image is generated. This new texture image of $1 / 8$ resolution with respect to the original one, is oriented to contribute to image segmentation, mainly complementing the information provided by the contours and solving the conflicts produced by the contours lack of continuity.
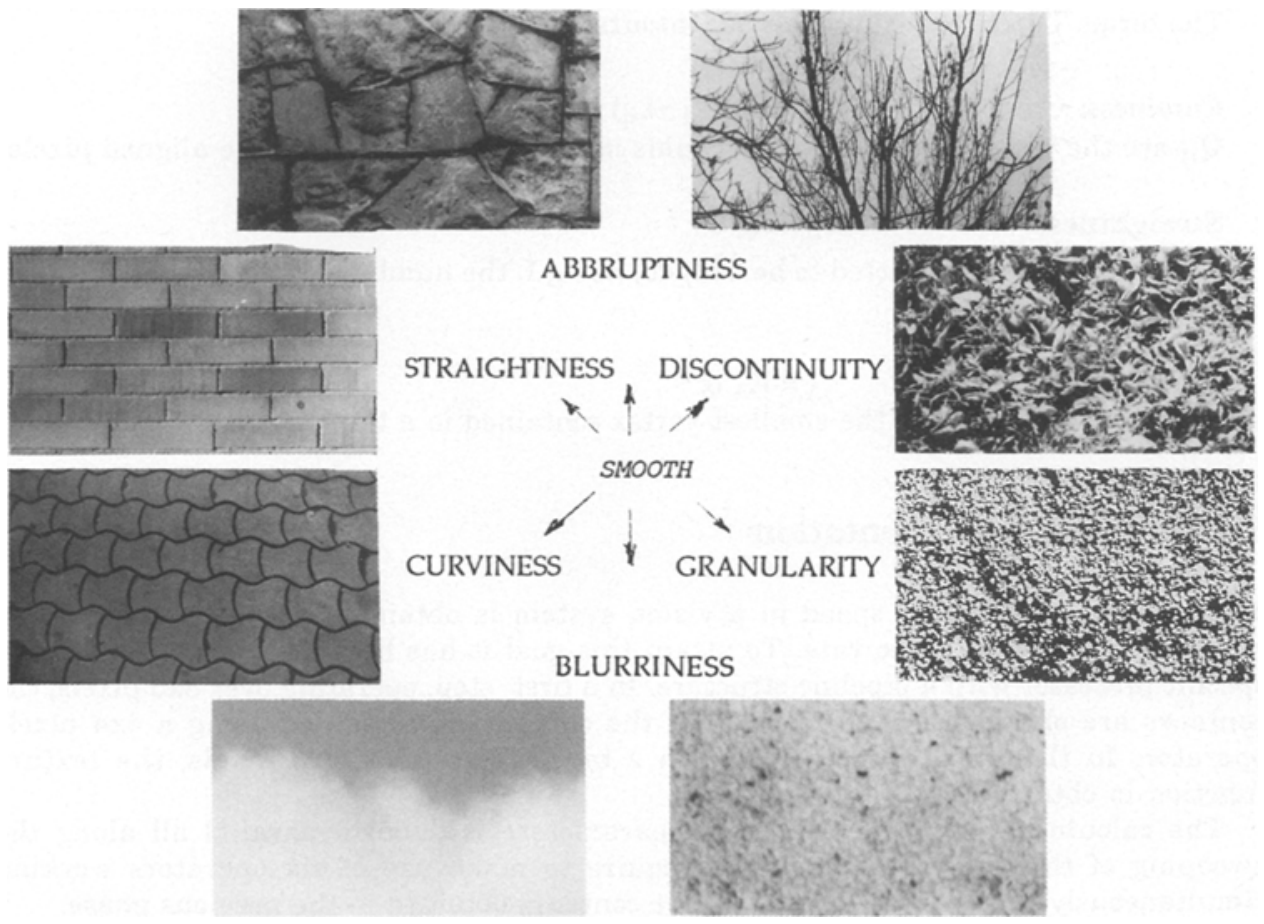

Fig. 1. The six texture parameters defined 


\section{Quantification Algorithms}

The defined parameters are quantified both by their module and their argument, that correspond to the main direction of the pattern considered, except the parameters blurriness and granularity that are considered isotropic.

The six parameters $\tau_{i}$ are quantified by applying to each $8 \times 8$ pixels, organized in 4 subregions of $4 \times 4$ pixels size, the following algorithms:

Blurriness:

$$
\tau_{\mathrm{B}}=\mathrm{K}_{\mathrm{B}}-\frac{(\mathrm{A}-\mathrm{D})+(\mathrm{C}-\mathrm{B})}{1+\Sigma \mathrm{P}_{\mathrm{ij}}}
$$

where $A, B, C$ and $D$ are the average gray levels corresponding to the four subregions what constitute a texel, and $\mathrm{P}_{\mathrm{ij}}$ are the pixels belonging to a contour within this region.

Granularity: $\quad \tau_{\mathrm{G}}=\mathrm{K}_{\mathrm{G}} \Sigma \mathrm{Q}_{\mathrm{ij}}$

being $Q_{i j}$ the pixels contained in the $8 \times 8$ region, which are detected as contours but in chains of length $\mathrm{L}<2$.

Discontinuity: $\quad \tau_{3 \mathrm{D}}=\mathrm{K}_{\mathrm{D}} \Sigma \mathrm{T}_{\mathrm{ij}}$

The terms Tij are the end pixels of contours of length $L>2$ in a texel

Curviness: $\quad \tau_{\mathrm{C}}=\Sigma\left(\mathrm{Q}_{\mathrm{ij}}-\mathrm{L}_{\mathrm{ij}}\right)$

$\mathrm{Q}_{\mathrm{ij}}$ are the pixels concatenated in chains of lenght $>3$ and $L_{\mathrm{ij}}$ are the aligned pixels.

Straightness: $\quad \tau_{L}=K_{L} L$

The value of $\tau_{L}$ is restricted to be $0<\tau_{L}<8$, being $L$ the number of aligned pixels within a texel. $(5<\mathrm{L}<8)$.
Abruptness:
$\tau_{\mathrm{A}}=\mathrm{K}_{\mathrm{A}} \boldsymbol{\alpha}$

where $\alpha$ is the angle of the smallest vertex contained in a texel.

\section{Hardware Implementation}

The maximum operation speed in a vision system is obtained when each processing phase is executed at video rate. To attain this goal it has been necessary to design a specific processor with a pipeline structure. In a first step, operating over $3 \times 3$ pixels, the contours are obtained; In a second step, the contours are thinned using a 4x4 pixels operator; In the third one, operating in a bigger size area, $8 \times 8$ pixels, the texture function is obtained.

The calculus of each one of the six parameters is done in parallel all along the sweeping of the image, but it would require to make use of six operators working simultaneously over the same data, ie., the contours obtained in the previous phase.

The implementation of the logical function to be carried out in this step requires some considerations in order to be physically feasible. Since the operator size is $8 \times 8$, the combinatory complexity is $2^{64}$, that's the reason why it has been decomposed into four 
parts, performing each one separately a function of complexity $2^{16}$, which can be already implemented with a single memory module. Fig. 2.

The decomposition of the texel in four quadrants presents some restrictions since they can not interchange information, but it allows to perform some global operations over an $8 \times 8$ environment, as it happens in the process of straight lines detection where both, the straight line slope and its continuity between adjacent subregions can be analyzed.

The processor operation time will be the time required to obtain the six texture parameters and that required to process these data. The six parameters are obtained at video rate, but with a delay of $3+4+8$ pixels. The algorithms used to process the texture parameters depend on the complexity of the scene with a range from 20 to $60 \mathrm{~ms}$.

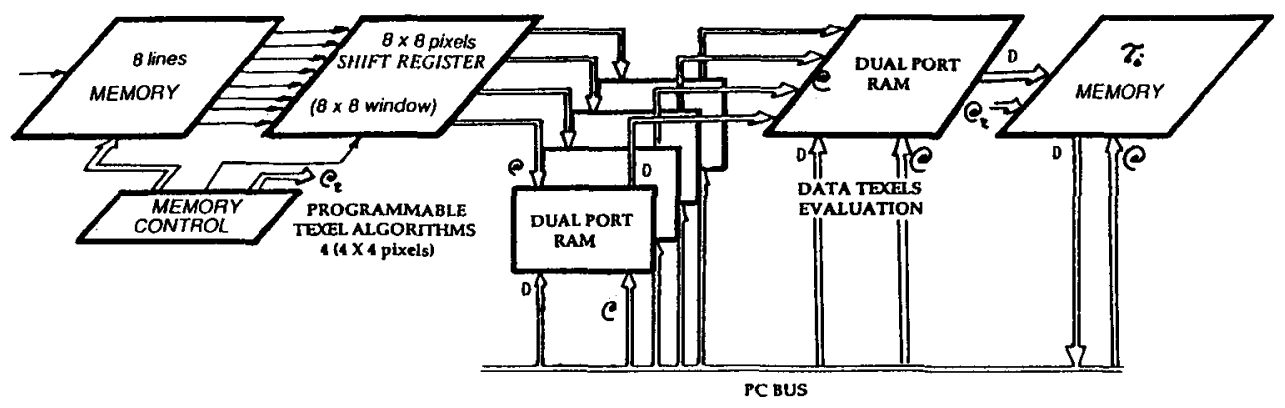

Fig. 2. Architecture of the processor designed to measure in real time the parameters $\tau_{i}$

\section{Results}

The texture images obtained from images of $256 \times 256$ pixels, having each texel a size of $8 \times 8$ pixels, result in a much lower resolution, $32 \times 32$ texels. In spite of the low resolution of the texture function generated, the results obtained are considered satisfactory for image segmentation of scenes in structured environments. The segmentation of the image is done based on the circumscribed contour of the elements of the scene, and the texture information is used to differenciate the regions.Fig. 3 shows the results obtained applying successively the straightness (3b). granularity (3c) and blurriness (3d) operators. These results can still be improved using color as complementary information for scene segmentation.

\section{References}

[Bou] Bouman, C., Liu, B.: Multiple Resolution Segmentation of Textured Images. IEEE Trans PAMI Vol. $13 \mathrm{n}^{22}$, (1991)

[Bro] Brodatz, P.: Textures. New York, Dover (1966) 

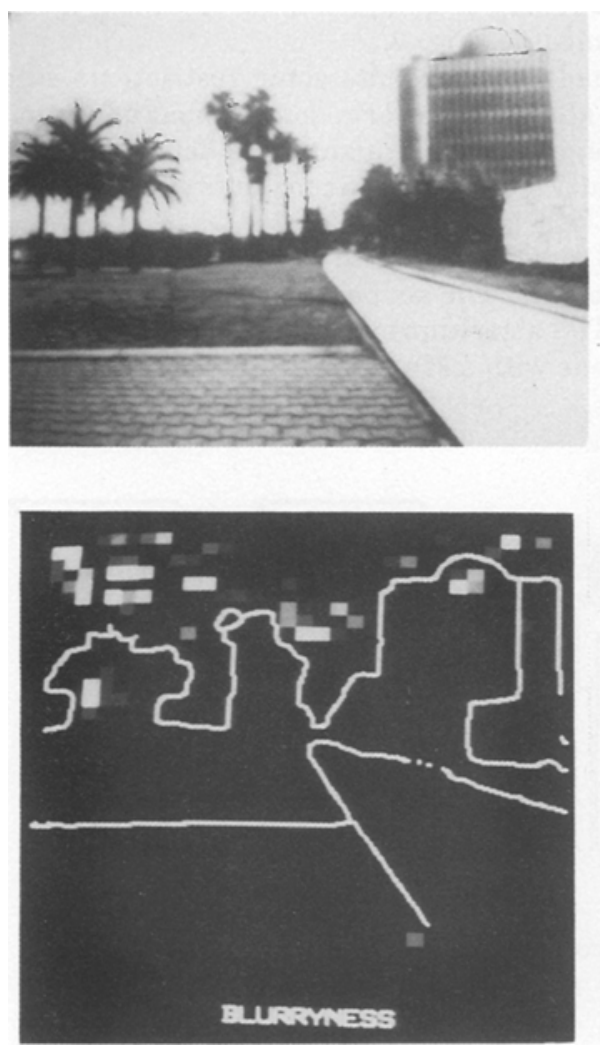
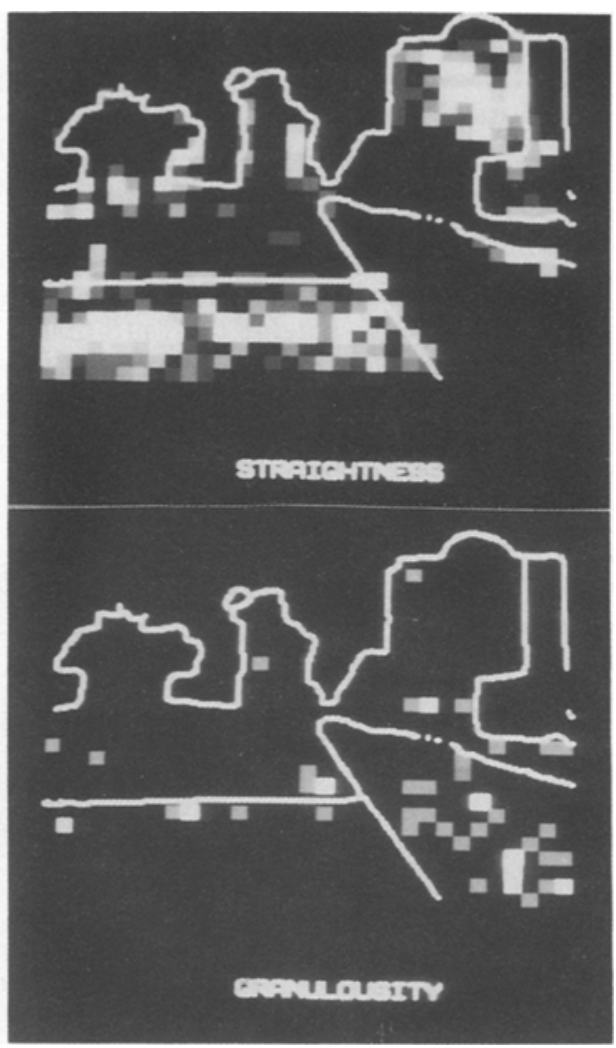

Fig. 3. Visualization over the circumscribed contours of the image shown in a) of the parameters: straightness b), blurriness c), and granularity d)

[Buf] du Buf, J.M.H. et al.: Texture Feature Performance for Image Segmentation. Pattern Recognition, vol.23 No.4 291-309 (1990).

[Cas] Casals, A. and Pagès, J.: A Vision System for Agricultural Machines Guidance. IARPW on Robotics in Agriculture and the food Industry. (1990).

[Har] Haralick, R.M.: Statistical and Structural Approaches to Texture. Proc. Int. Joint Conference on Pattern Recognition, vol.4 45-69, Kyoto (1978)

[Man] Manjunath, B.S. and Chellappa, R.: Unsupervised Texture Segmentation Using Markow Randow Field Models. PAMI vol.13 No.5 (1991)

[Mat] Matsuyama et al.: A structural Analyzer for Regularity Arranged Textures. Computer Graphics and Image Processing 18 259-279 (1982)

[Shi] Shipley, T. and Shore, T.: The Human Texture Visual Field: Fovea-toPeriphery Pattern Recognition. Pattern Recognition, vol.23 No.11 (1990).

[Tsu] Tsuji, S. and Tomita F.: A Structural Analyzer for a Class of Textures. Computer Graphics and Image Processing 2 216-231 (1973)

[Zuc] Zucker, S.W.: Toward a Model of Texture Computer Graphics and Image Processing 5 190-202 (1976) 